\title{
Electric Filed Simulation and Structure Optimization for 40.5kV GIS Based on Finite Element Method
}

\author{
Xian $\mathrm{CHENG}^{1,2}$, Jianying $\mathrm{ZHONG}^{2}$,Fang $\mathrm{YANG}^{2}$, Zhanqing $\mathrm{CHEN}^{1}$, Duanlei \\ $Y_{U A N}^{2}$, Ming PAN ${ }^{2}$ \\ ${ }^{1}$ School of Electrical Engineering, Zhengzhou University, Zhengzhou 450000, China; \\ ${ }^{2}$ Ping Gao Group Co., Ltd, Pingdingshan 467000, China \\ chenxian9@163.com
}

Keywords: GIS gas chamber; electric-filed calculation, electrostatic field; structure optimization

\begin{abstract}
The electric field distribution in the Gas insulated switchgear (GIS) gas chamber is an important factor for the design of GIS structure. The electric field distribution in the GIS gas chamber should be uniform as far as possible, to avoid excessive concentration of the electric field causing the occurrence of flashover and discharge in GIS. The electric field intensity in the gas chamber is higher than its dielectric's isolating strength when SF6/N2 mixed gas is used as the insulation dielectric of the gas chamber to reduce the consumption of pure SF6, and the research in this paper aim to solve this problem. Electric-filed numerical analysis and optimal design of GIS gas chamber structure have been done in this paper. The GIS gas chamber 3D model is built in Solidworks, and finite element analysis of the model is calculated in ANSYS. Electric field numerical calculation for the gas chamber model is calculated based on the theory of electrostatic field. The calculation results show that the electric field intensities of isolated switch, earthing switch and insulation rod are excessive high, and the optimizations for its internal structure are necessarily followed, then calibrating calculations of electric field are carried on the optimized model. The calculating results convey that the electric field intensity in gas chamber decreased significantly, and the maximum value of electric field intensity is lower than the dielectric strength of alternative gas. It means that the alternative gas can meet the insulation requirements for the gas chamber after optimization.
\end{abstract}

\section{Introduction}

With the continuous development of the power system, the gas insulated switchgear (GIS) which is known as reliable and compact apparatus has become an integral unit for the distribution network. The electric field distribution in its gas chamber is an important content for the structure design of GIS. Favorable design of its electric field distribution should be uniform as far as possible, to avoid excessive concentration of electric field which could lead to the flashover and discharge in GIS. High cost and long time-consuming of the high voltage experiments have limited the efficiency and cost of high voltage apparatus' development. With the prevalence and development of numerical simulation theory and large commercial software analysis for electromagnetic field, simulating three-dimensional electric field distribution becomes possible [1]. Electric field numerical simulation applied to the design of new GIS gas chamber can effectively reduce the development costs and shorten the development period.

At present most of the GISs adopt pure SF6 gas as insulating medium. SF6 gas has an excellent insulating property, but the usage of SF6 would be controlled before 2020 in the world because of its severe greenhouse effects [2-4]. It is found that the insulating strength of nitrogen can increase greatly by mixing up with small amount of SF6 [5]. And the electric strength of 30\%SF6/70\%N2 is $5.5 \mathrm{kV} / \mathrm{mm}$ at $0.15 \mathrm{MPa}$, slightly lower than pure SF6 gas at the same pressure. The mixed gas SF6/N2, a kind of environmentally friendly gas, used as the electrical insulating medium in GIS instead of pure SF6 gas is one of the most effective methods to reduce the usage of SF6 gas according to the previous studies. This paper studies the phenomenon that the electric field intensity in the gas chamber would get higher than the dielectric's insulating strength when taking SF6/N2 mixed gas as 
insulation medium instead of pure SF6. The regions which noted with high electric field strength in the gas chamber have been found out with the numerical simulation work of electric field while taking the gas chamber structure as studying object, and the electric field strength in the gas chamber has been decreased through the optimization of its structure. All the works mentioned above can provide basic research references for the application of environmentally friendly gas in 40.5kV GIS.

\section{Gas chamber model and electric field calculation}

Gas chamber model. The gas chamber components mainly include busbar, busbar insulation tubings, vacuum interrupters and insulation drawbars, 3 position switchgear, connecting parts, etc. The calculating areas are composed of these components and the insulation dielectric filled in the gas chamber, and the gas chamber shell is the calculating boundary. The 3D model can be more accurate to reflect the characteristics of gas chamber model, because most of the gas chamber components are non-symmetrical structure. Gas chamber $3 \mathrm{~d}$ model is established according to the actual size, as shown in fig1.

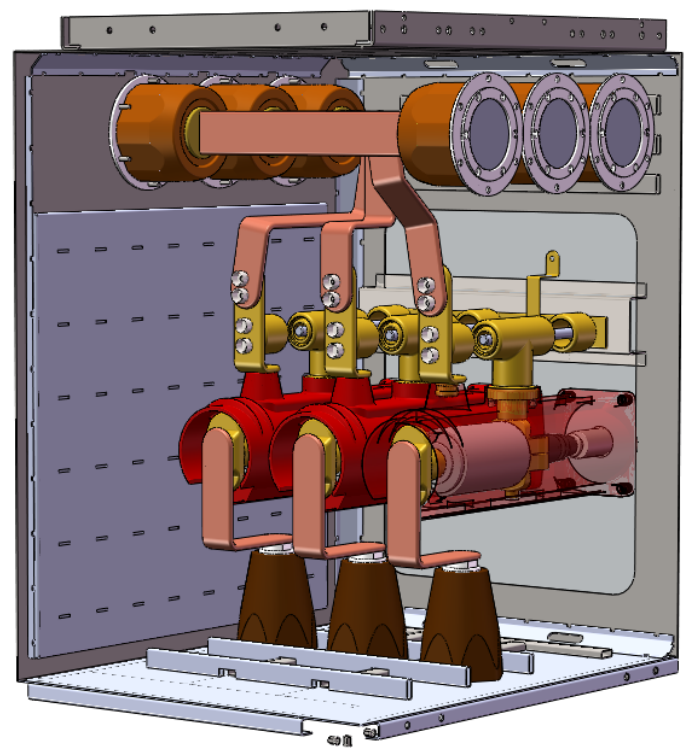

Fig.1 Gas chamber model

The mathematic model of electric field. There are many materials in the gas chamber, mainly including conductors and insulation materials. Conductor material is copper which would be regarded as equipotential body in the electric field calculation for its good electrical conductivity. Insulating materials include PVC, SF6/N2 mixed gas, epoxy resin, etc., and the displacement currents of these materials are much higher than the conduction current under the effect of frequency electric field, so these insulating materials can be regarded as ideal dielectrics. The influences of induced electric field can be neglected because of the low frequency of power frequency voltage. The electric field in the gas chamber can be considered as electrostatic field, and the distribution of electric field is expressed by electrostatic field equation. Electrostatic field equation of differential form can be represented as:

$$
\begin{aligned}
& \nabla \bullet D=\rho \\
& \nabla \times E=0
\end{aligned}
$$

In combination with constitutive equation:

$D=\varepsilon E$

And these three equations constitute basic equations of the static electric field, D is the electric displacement; $\rho$ is the charge density; $\mathrm{E}$ is the electric field intensity; $\varepsilon$ is the dielectric constant determined by the material properties; and the electric field intensity vector is equal to negative potential gradient: 
$E=-\nabla \varphi$

Simultaneous equations.

$$
\nabla^{2} \varphi=-\frac{\rho}{\varepsilon}
$$

Equation(5) is the differential equation of electrostatic potential. The potential value of any point in the space can be calculated out by solving the equation. The distribution of electrostatic field can be determined with consideration of boundary conditions. The boundary conditions electrostatic field can be expressed as:

$$
-\varepsilon_{1} \frac{\partial \varphi_{1}}{\partial \mathrm{n}}+\varepsilon_{2} \frac{\partial \varphi_{2}}{\partial \mathrm{n}}=\sigma
$$

$\sigma$ is free charge surface density.

The electric field calculation. The gas chamber model has been meshed before electric field calculation. The mesh quality has a significant effect on the simulation results. This paper adopts tetrahedral grid to mesh the gas chamber model, and the meshing result is shown in fig2. The gas chamber model is meshed into 7245180 nodes finally, and it can meet the requirements of the calculation accuracy.
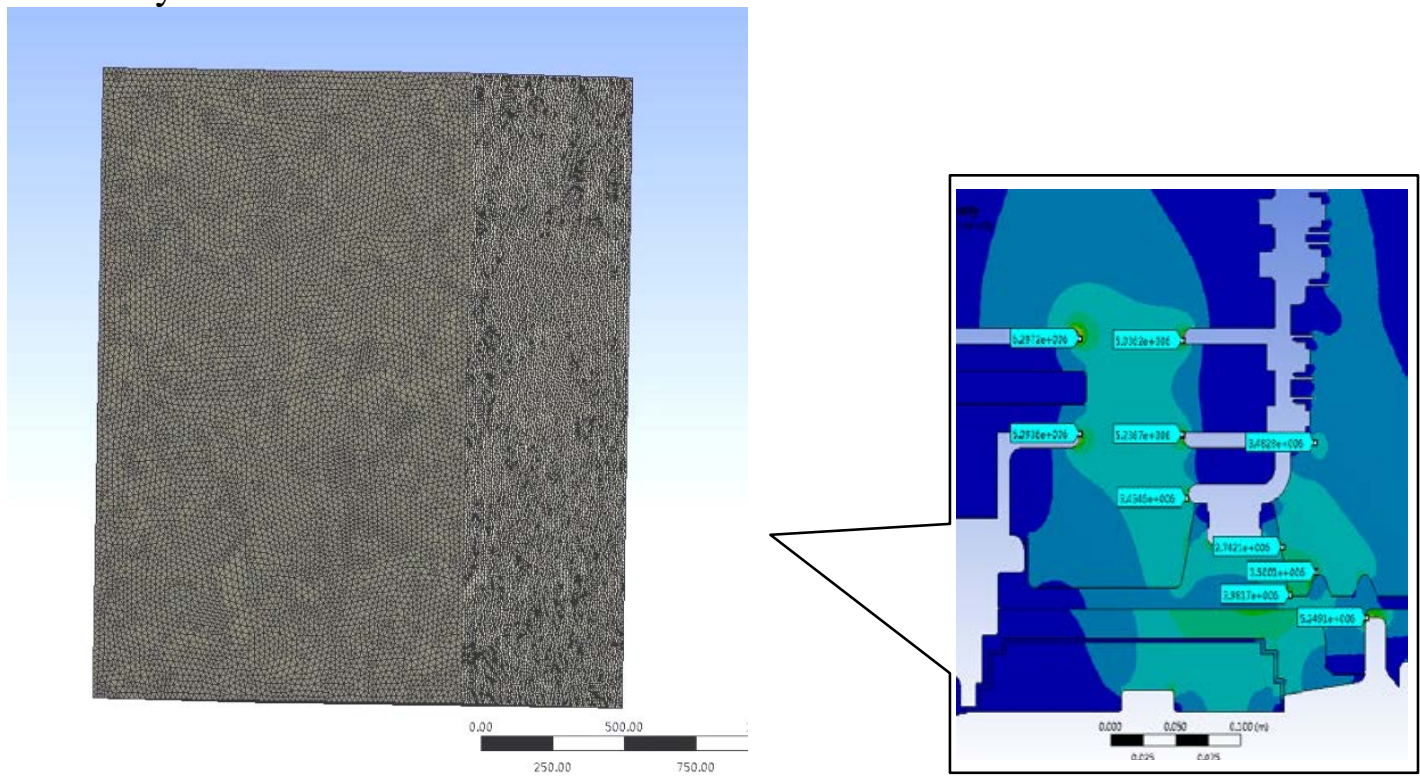

Fig.2 Meshing

The $40.5 \mathrm{kV}$ GIS is required to endure the lightning impulse voltage at $215 \mathrm{kV}$ for isolation fracture and $185 \mathrm{kV}$ for ground fracture. The isolation fracture is applied with voltage at $215 \mathrm{kV}$ while the voltage on ground fracture is $185 \mathrm{kV}$ in the simulation work, and the ground contact and the shell are kept at zero potential. Fig 3 is the electric field intensity distribution of the gas chamber when the isolation switches work at the grounding position. Fig 4 is the electric field intensity distribution of the gas chamber when the isolation switches work at the operating position. As shown in the diagram, the regions with maximum electric field intensity appear in the end of the isolated fracture when the isolated switch is working at the grounding position. The value of the maximum electric field has exceeded the alternative gas's electric strength. The electric field intensity distributions on ground fracture and insulation pole surface are relatively concentrated and the value of the maximum electric field has exceeded the alternative gas's electric strength too. So the electric field distribution on above mentioned regions cannot meet the insulating requirements of the gas chamber, and the gas chamber structure need to be optimized then. 


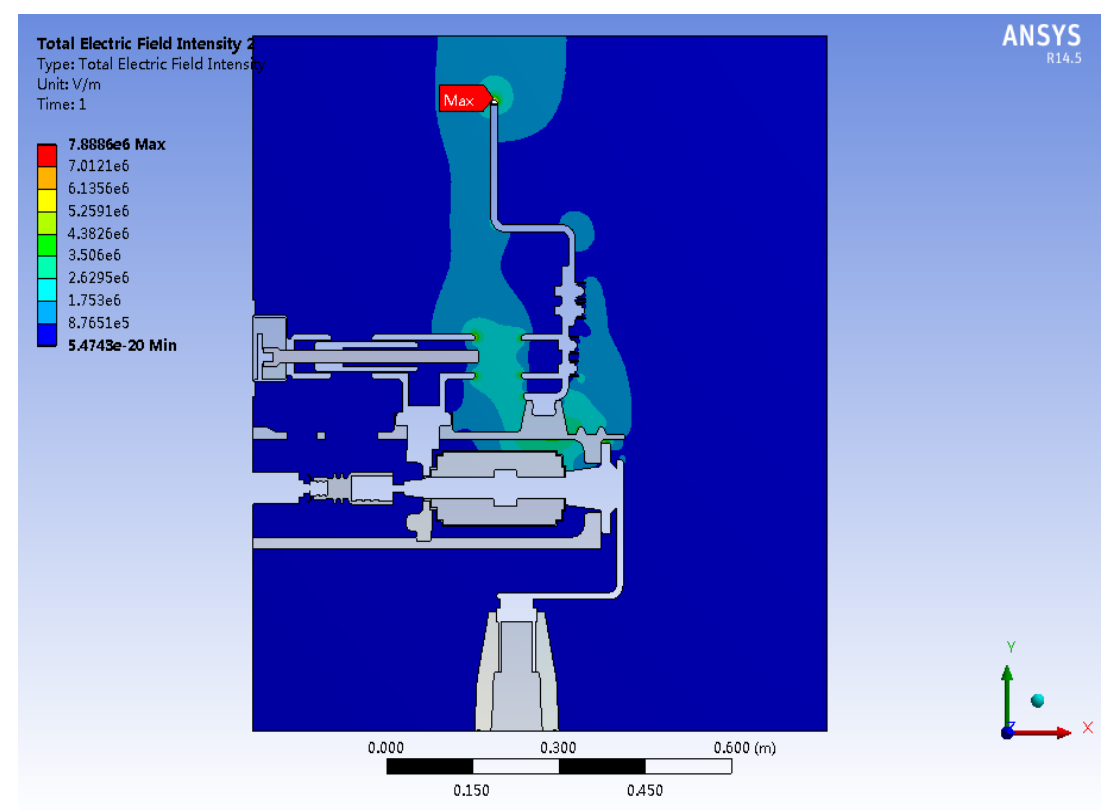

Fig.3 Electric field intensity of isolated fractures

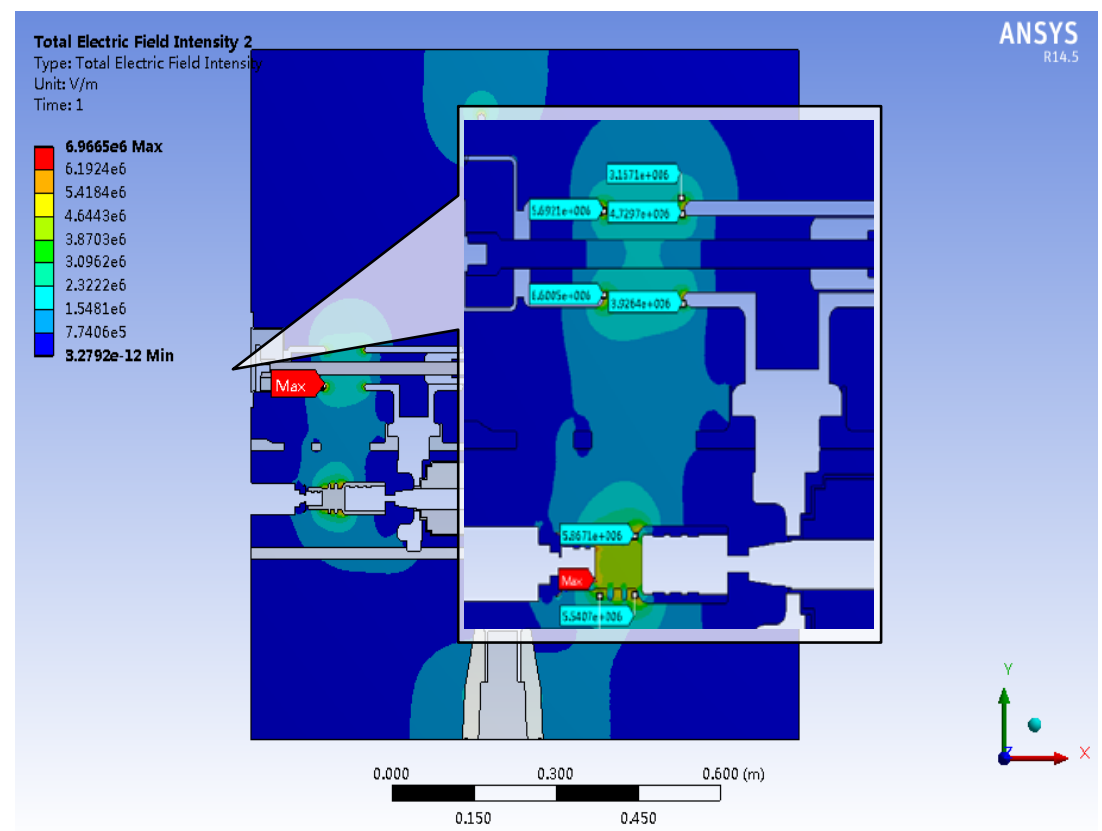

Fig.4 Electric field intensity of grounded fractures

\section{Structure optimization and electric field checking calculation of the gas chamber}

Structure optimization. The structural optimization for the high electric field intensity areas mainly include the added shielding devices on three position switch, the modified and improved structure of the terminal and connecting terminal, and redesigned the insulation drawbars. The gas chamber model is reestablished after the optimization, as shown in fig 5. 


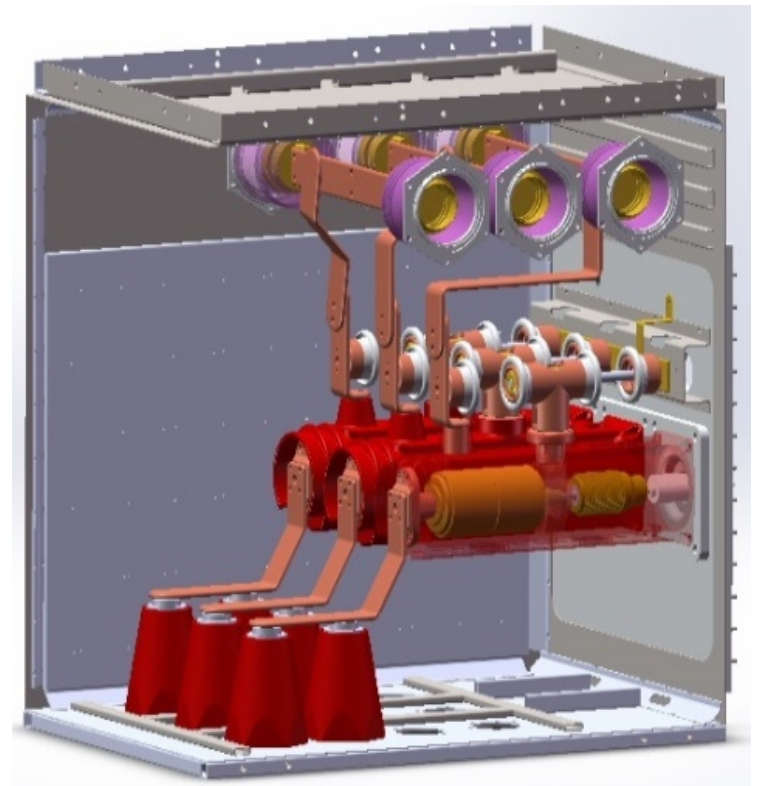

Fig.5 The model of the gas chamber after optimization

Electric field calibration. Electric field calculating work on the optimized gas chamber model is done, and the calculating steps are the same as before. The results of calculation are shown in fig 6 and fig7. Drawing from the results, the electric field distribution patterns are similar to the previous simulating results.. The electric field intensities on the ground fracture, isolation fracture and the surface of insulating rod are still high. But the maximum value of electric field strength is below $5.5 \mathrm{kV} / \mathrm{mm}$. The insulating strength of alternative gas can meet the gas chamber's insulating requirements completely.

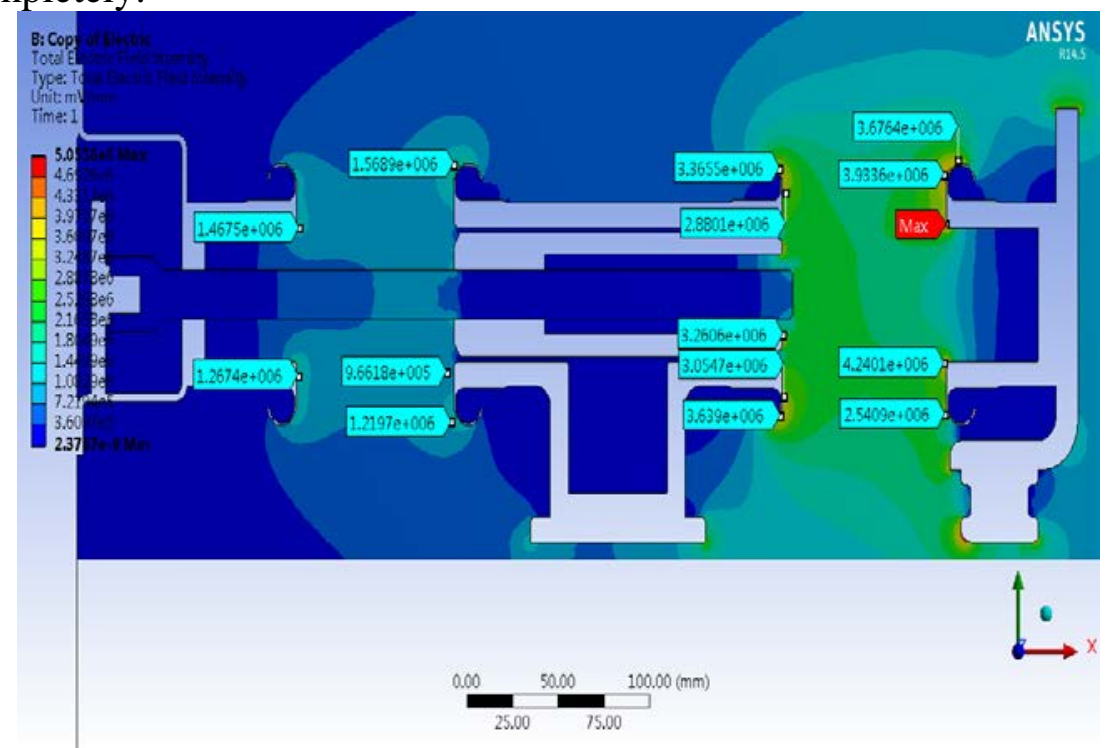

Fig.6 Electric field intensity of isolated fractures after optimization 


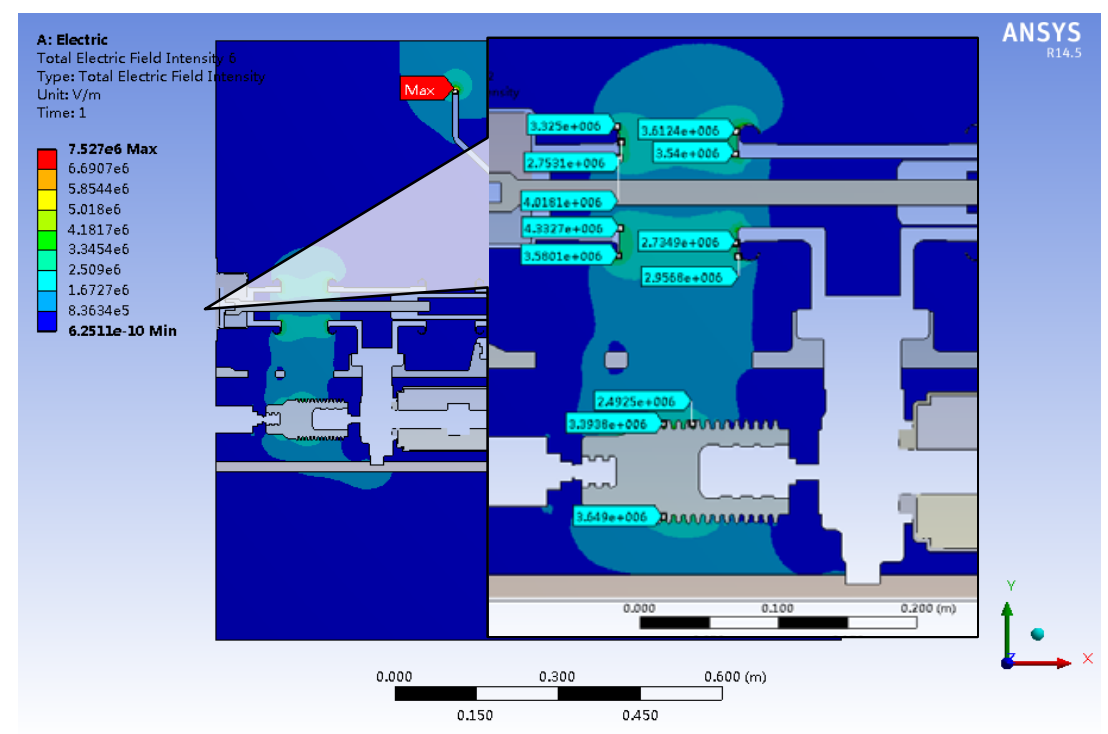

Fig.7 Electric field intensity of grounded fractures after optimization

Table1: Comparison of before and after optimization

\begin{tabular}{|c|c|c|c|}
\hline Optimization part & Before optimization & After optimization & $\begin{array}{c}\text { Whether to meet the } \\
\text { requirements }\end{array}$ \\
\hline Isolated fracture & $6.29 \mathrm{kV} / \mathrm{mm}$ & $4.24 \mathrm{kV} / \mathrm{mm}$ & $\mathrm{Y}$ \\
\hline Grounded fracture & $6.60 \mathrm{kV} / \mathrm{mm}$ & $4.33 \mathrm{kV} / \mathrm{mm}$ & $\mathrm{Y}$ \\
\hline Insulation drawbars & $5.86 \mathrm{kV} / \mathrm{mm}$ & $3.65 \mathrm{kV} / \mathrm{mm}$ & $\mathrm{Y}$ \\
\hline
\end{tabular}

\section{Conclusion}

This paper studies the problem that the electric field intensity of the gas chamber can't meet the insulation requirements when SF6/N2 mixed gas is used as the insulation medium of the gas chamber instead of pure SF6. The maximum electric field strength of the gas chamber is reduced with numerical simulation of electric field, distribution of the field strength and optimization of the gas chamber structure. The electric field intensity of the whole gas chamber is less than $5.5 \mathrm{kV} / \mathrm{mm}$, ensuring the insulation strength of the substitute gas could meet the insulation requirements of the gas chamber.

\section{Acknowledgement}

This work was supported by National Nature Science Foundation of China (No.51407163, No.51477024) and China Postdoctoral Science Foundation (No. 2014M552012, No.2015T80778).

\section{References}

[1] Xian Cheng, Minfu Liao, Xiongying, Duan; Jiyan, Zou. Static Electric Field Distribution of Hybrid Circuit Breaker Based on Ansoft. XXVth International Symposium on Discharges and Electrical Insulation in Vacuum, Tomsk, Russia, 2012, pp94-91.

[2] The Working Group III. Intergovernmental panel on climate change: Fifth Assessment Report. Geneva, Switzerland: The Intergovernmental panel on climate change, 2014. 
[3] Xian Cheng, Minfu, Liao; Xiongying, Duan; Jiyan, Zou. Simulation Study on Interaction between Vacuum Switch and SF6 Switch in the High-Current Interruption Process. Journal of Computational Information Systems. Vol.8, No.11, pp.4735-4742, 2012.

[4] Xian, Cheng, Minfu, Liao; Xiongying, Duan; Jiyan, Zou. The Voltage Distribution Characteristics of Hybrid Circuit Breaker during High Current Interruption. Plasma Science and Technology. Vol.15, No.8, pp.800-807, 2012.

[5] Christophorou L. G, and Van Brunt R. J. SF6/N2 Mixtures: basic and HV insulation properties. IEEE Transactions on Dielectrics and Electrical Insulation, Vol.2, No.5, pp.952-1003, 1995.

[6] Wenjian Yu, Zeyi Wang, and Xian long Hong. Preconditioned multi-zone boundaryelement analysis for fast 3D electric simulation. Engineering Analysis with Boundary Elements, Vol.28, pp 1035-1044, 2004.

[7] Jianyuan XU, Chunwei REN, Binge Si, et al. Three dimensional electric filed analysis of $40.5 \mathrm{kV}$ SF6 cubicle gas-insulated metal-enclosed switchgear. Proceedings of the CSEE, Vol.28, No.15, pp.136-140, 2008.

[8] Zhigang Liu, Xin Lin, Yingsan Geng. The calculation and analysis of three-dimension electric field of SF6 tank-type circuit breaker. High Voltage Engineering, Vol.29, No.3, pp 27-30, 2003.

[9] Takuma T, Kawamoto $\mathrm{T}$. Numerical calculation of electric fields with a floating conductor. IEEE Trans. on Dielectrics and Electrical Insulation, Vol.4, No2, pp 177-188, 1997.

[10]BOECK WGRAFRFINKEL M. Effect of surface roughness and curvature on Streamer Inception and breakdown of N2/SF6mixtures. 7th ICPADM. Nagoya, 2003, pp 105-108.

[11]Aiqing Ma, Chuan Cai, Xiuchen Jiang, et al. Software package of electric field intensity and potential distribution of SF6 circuit breaker in GIS. High Voltage engineering, Vol.33, No.6, pp 106-109, 2007.

[12] Yueming WANG, Lingfu KONG.. Magnetic properties study of electromagnetic flow meter based on ANSYS. Journal of Computational Information Systems, Vol.7,No.8, pp 2779-2786, 2011. 